\title{
Terapia de resincronización en pacientes con insuficiencia cardiaca: experiencia acumulada de 10 años
}

\author{
ANDRÉS ENRÍQUEZ, RAÚL BARRERO, ALEX BITTNER, \\ PATRICIA FRANGINI, MARIANA BAEZA, ISLANDIA MILLAPÁN, \\ ROLANDO GONZÁLEZ, ISMAEL VERGARA
}

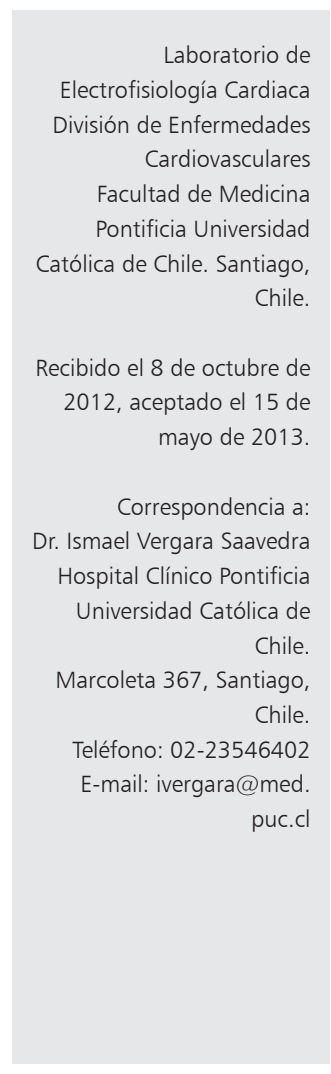

\section{Cardiac resynchronization therapy in patients with heart failure: A 10-year experience}

Background: Multiple randomized trials support the clinical benefits of cardiac resynchronization therapy (CRT) in patients with heart failure (HF) and ventricular dyssynchrony. Since the year 2000 this therapy has been increasingly used in Chile. Aim: To describe the clinical characteristics and follow-up of HF patients undergoing CRT in a single Chilean university hospital during the last 10 years. Patients and Methods: All patients undergoing CRT between 2000 and 2010 in our university hospital were included. Clinical and echocardiographic data were extracted from medical records and mortality causes were obtained from the National Identification Service. Results: A total of 252 patients underwent CRT during the study period. Seventy five percent were in New York Heart Associatin (NYHA) functional class III and mean ejection fraction was $29 \pm 10 \%$. Complete left bundle branch block was present in 55\% and 20\% had permanent atrial fibrillation (AF). Mean survival was $86 \%$ at 1 year and $82 \%$ of patients in NYHA class III-IV improved at least one functional class. Survival was poorer in patients with ischemic etiology (hazard ratio (HR) 1.48), functional class IV (HR 2.2), right bundle branch block (RBBB) (HR 3.1) and $A F$ (HR 3.4). No survival differences were observed between patients with and without an implanted cardiodefibrillator. Conclusions: This series show good clinical outcomes, comparable to those reported in randomized trials. Predictors of worse survival included an ischemic etiology, functional class IV, RBBB and AF. Patients with a defibrillator had no better survival, which could be relevant in countries with limited health care resources.

(Rev Med Chile 2013; 141: 968-976).

Key words: Cardiac resynchronization therapy; Heart failure; Mortality.

$\mathrm{P}$ ese a los avances en el conocimiento fisiopatológico de la insuficiencia cardiaca (IC) y al desarrollo de nuevas terapias, su manejo sigue siendo complejo, con altas tasas de hospitalización asociadas, pobre calidad de vida y elevados costos de salud ${ }^{1}$. Incluso con terapia médica óptima, la IC avanzada se asocia a una tasa de mortalidad anual $>10 \%^{2}$ y alrededor de $50 \%$ a 5 años.

En pacientes con IC es frecuente la presencia de trastornos de conducción intraventricular ${ }^{3}$, en especial bloqueo completo de rama izquierda (BCRI). Este trastorno se traduce en un patrón anormal de activación del ventrículo izquierdo (VI), con contracción tardía de la pared lateral respecto al septum, y la pérdida de la sincronía ventricular conduce a deterioro progresivo de la función contráctil. Basado en las consecuencias fisiopatológicas de la asincronía electromecánica ha surgido la estimulación cardiaca mediante 
Terapia de resincronización en insuficiencia cardiaca - A. Enríquez et al

marcapasos biventriculares como tratamiento en pacientes con IC refractaria al tratamiento farmacológico.

La evidencia acumulada de numerosos estudios clínicos con distribución aleatoria ha demostrado que la terapia de resincronización cardiaca (TRC) se asocia consistentemente a mejoría sintomática y reducción de las hospitalizaciones en pacientes con IC clase New York Heart Association (NYHA) IIIIV, fracción de eyección VI (FEVI) $\leq 35 \%$, y QRS $>120 \mathrm{~ms}^{4-9}$. El estudio COMPANION ${ }^{9}$ demostró además un aumento de sobrevida cuando el resincronizador se asoció a desfibrilador implantable y el CARE-HF demostró 36\% de reducción de mortalidad en el grupo tratado con resincronizador versus terapia médica óptima². Más recientemente los resultados del MADIT-CRT ${ }^{10}$ y REVERSE ${ }^{11}$ han demostrado que los beneficios de la TRC son extensibles a pacientes con IC leve.

En el Hospital Clínico de la Pontificia Universidad Católica de Chile la TRC está disponible desde el año 2000. El objetivo del presente estudio es describir los resultados inmediatos y a largo plazo en pacientes sometidos al implante de un marcapaso resincronizador en los primeros 10 años de su implementación.

\section{Métodos}

Se incluyeron todos los pacientes sometidos al implante de un marcapaso resincronizador en nuestro centro entre los años 2000 y 2010.

Se analizaron las características clínicas, electrocardiográficas y ecocardiográficas de los pacientes, los resultados inmediatos del implante y las complicaciones intrahospitalarias.

Durante la intervención se realizó inicialmente el implante del electrodo ventricular derecho de fijación activa, prefiriendo la posición septoinfundibular o septal media. Posteriormente, se realizó canulación del seno coronario, utilizando catéteres preformados o deflectables, sobre los cuales se avanzó el catéter guía. Una vez asegurado el acceso al seno coronario, se realizó venografía para seleccionar las ramas venosas adecuadas para avanzar el electrodo. Siempre que fue posible se seleccionó una rama posterolateral o lateral, evitando las posiciones anteriores. Se avanzó el electrodo (unipolar o bipolar) hacia la vena mediante una guía rígida o sobre guía de 0,014 ". Una vez posicionado el electrodo se buscó umbrales de estimulación satis- factorios (menores a 2,0 V a $0,5 \mathrm{~ms}$ ), con ausencia de estimulación frénica a amplitud máxima $(10 \mathrm{~V}$ a $0,5 \mathrm{~ms}$ ). En caso de no cumplirse estos requisitos, se buscó posiciones alternativas hasta agotar las opciones. Finalmente, se implantó el electrodo auricular, de fijación activa, para proceder luego al retiro del sistema de implante del electrodo de seno coronario. Se midió la duración del QRS en las doce derivaciones del electrocardiograma registradas en el polígrafo (Prucka Engeneering), inmediatamente antes y posterior al implante.

Todos los pacientes tuvieron seguimiento clínico y control del resincronizador en la Clínica de Marcapasos de nuestra institución cada 3-6 meses. En la mayoría se realizó optimización del intervalo auriculoventricular e interventricular bajo técnicas ecocardiográficas durante los primeros 7 días postimplante. Los datos relativos a mortalidad y causas de muerte fueron obtenidas de las fichas clínicas y del Servicio de Registro Civil.

Las características de la población se expresaron como promedios \pm desviación estándar para las variables continuas y como porcentajes para las variables categóricas. El análisis estadístico se realizó mediante test t-Student para las variables cuantitativas, $\chi^{2}$ para las variables cualitativas $y$ curvas de Kaplan-Meier con test de log Rank para el análisis de sobrevida.

\section{Resultados}

\section{Características de los pacientes}

Durante el período estudiado, 252 pacientes fueron sometidos al implante de un marcapaso resincronizador. Setenta y cinco por ciento eran hombres y la edad promedio fue $68,5 \pm 11,4$ años (16-91 años), con $67,7 \%$ de pacientes mayores de 65 años y 16,3\% mayores de 80 años. La FEVI era de $29 \pm 10 \%$ y el diámetro telediastólico del VI era de $67 \pm 10 \mathrm{~mm}$. Respecto a la etiología, la mayoría (51\%) tenía origen isquémico (Figura 1). La Figura 2 muestra la distribución de los pacientes según capacidad funcional (CF), con $75 \%$ en clase III. Setenta y cinco por ciento se trató de un primoimplante y $25 \%$ de un "upgrade" a partir de un sistema más simple (VVI o DDD) a un sistema biventricular. Cincuenta y cinco por ciento de los pacientes tenía BCRI, 11\% bloqueo completo de rama derecha (BCRD) y $9 \%$ tenía QRS angosto o alteraciones inespecíficas de la conducción intraventricular (Figura 3). Al momento del implante, 


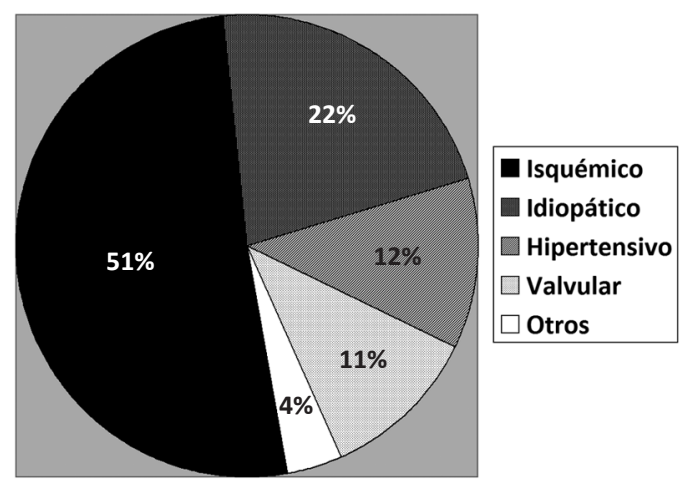

Figura 1. Distribución de los pacientes según etiología.

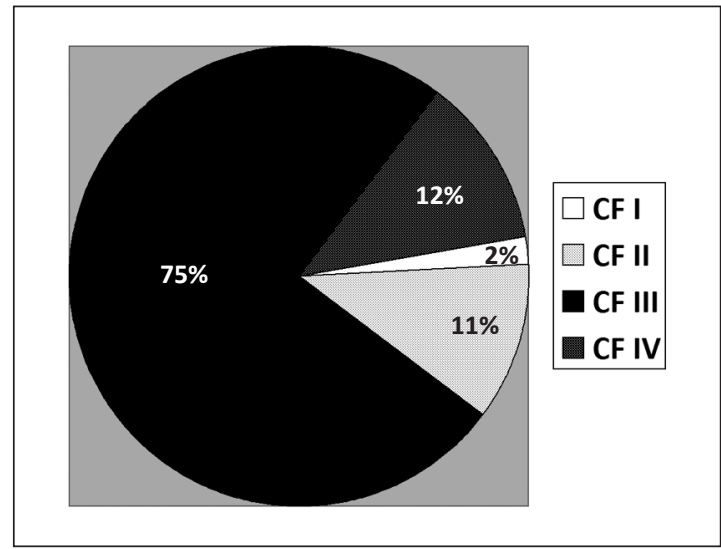

Figura 2. Distribución de los pacientes según clase funcional NYHA previo al implante del marcapaso resincronizador. El 87\% se encontraba en CF III O IV.

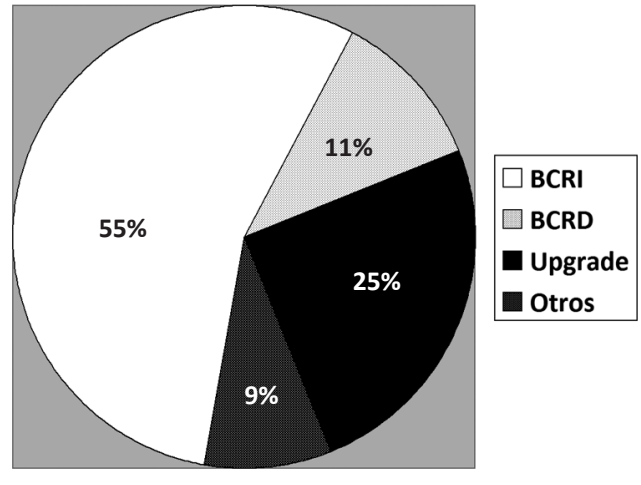

Figura 3. Morfología del QRS.
$81 \%$ de los pacientes estaba en ritmo sinusal y $19 \%$ presentaba una arritmia auricular permanente, predominantemente fibrilación auricular (FA). En estos últimos se selló el puerto correspondiente al electrodo auricular, y en $77 \%$ se realizó además ablación con radiofrecuencia del nodo AV para asegurar un alto porcentaje de estimulación biventricular. Treinta y dos por ciento de los pacientes recibió además un cardiodesfibrilador implantable (CDI), correspondiendo $73 \%$ de éstos a prevención primaria y $27 \%$ a prevención secundaria.

\section{Resultados inmediatos}

Se logró el implante exitoso en 235 pacientes (93,3\%), posicionándose el electrodo preferentemente en una vena coronariana posterolateral o lateral, lo cual se logró en $91 \%$, y en una rama anterolateral, frente a ausencia de ramas más favorables, en $9 \%$ de los casos. El umbral de estimulación del electrodo de seno coronario fue 0,9 $\pm 0,5 \mathrm{~V}(0,2-3,1 \mathrm{~V})$. El tiempo de radioscopia fue $34,3 \pm 19 \mathrm{~min}$. Al analizar por separado la primera y la segunda mitad de los pacientes de la serie, observamos que la tasa de éxito en el implante fue significativamente mayor en los últimos 126 pacientes (90,0 versus $96,8 \%$ ).

Las causas de fracaso en el implante se enumeran en la Tabla 1, siendo las más frecuentes la imposibilidad de canular el seno coronario o sus ramas afluentes $(4,4 \%)$ y la disección del seno coronario (1,6\%). En 6 de estos pacientes se implantó un electrodo en VI por vía epicárdica.

\section{Complicaciones del implante}

No hubo mortalidad asociada al procedimiento. Hubo desplazamiento agudo del electrodo de

Tabla 1. Causas de fracaso en el implante del electrodo en seno coronario para estimulación ventricular izquierda

\begin{tabular}{|lc|}
\hline & n (\%) \\
$\begin{array}{l}\text { Imposibilidad de canular seno coronario o } \\
\text { vena coronariana }\end{array}$ & $11(4,4 \%)$ \\
\hline Disección de seno coronario & $4(1,6 \%)$ \\
Umbral demasiado alto & $1(0,4 \%)$ \\
Ausencia de vena coronaria adecuada & $1(0,4 \%)$ \\
Total & $17(6,7 \%)$ \\
\hline
\end{tabular}


seno coronario en 9 casos $(3,6 \%)$, lo que obligó a reintervención y, como ya se dijo, disección del seno coronario en 4 casos (1,6\%). En 3 pacientes se observó estimulación frénica no solucionable con reprogramación, lo que motivó una reintervención $(1,2 \%)$. La tasa de complicaciones mayores fue de 2\%: neumotórax en 2 pacientes $(0,8 \%)$ y derrame pericárdico en 3 casos $(1,2 \%)$, de los cuales 2 requirieron pericardiocentesis por taponamiento cardiaco (atribuidos a disección del seno coronario). Además se produjo desplazamiento del electrodo auricular en 1 paciente $(0,4 \%)$ y hematoma moderado en 4 pacientes $(1,6 \%)$, sin necesidad de reoperación. No existió ninguna perforación cardiaca ni infección de herida operatoria.

\section{Eficacia clínica y seguimiento}

De los pacientes con implante exitoso, $82 \%$ mejoró en al menos una categoría de CF en forma sostenida. La tasa de respuesta clínica no difirió significativamente entre hombres y mujeres ni tampoco entre pacientes con etiología isquémica versus no isquémica (Figura 4). En cambio la tasa de respuesta fue significativamente mayor en pacientes en ritmo sinusal respecto a aquellos en FA permanente ( 86 vs $69 \%$, p 0,015). Con respecto a la morfología del QRS, los más beneficiados fueron los portadores de BCRI, seguido de aquellos con BCRD y los pacientes con upgrade $(87,7 ; 78,5 \mathrm{y}$ $70 \%$ respectivamente, $\mathrm{p} 0,013$ ).

La duración del QRS se redujo de $180 \pm 37 \mathrm{~ms}$ a $155 \pm 21 \mathrm{~ms}(\mathrm{p}<0,001)$. La Figura 5 muestra un ejemplo de los electrocardiogramas pre y post implante de un resincronizador, destacando una marcada reducción en la duración del QRS y aparición de onda $\mathrm{R}$ en precordiales derechas.

Respecto a la función cardiaca, después de un período promedio de 8 meses se observó una mejoría de la FEVI desde $28,5 \pm 10,7 \%$ a $31,8 \pm 11,8 \%$ $(\mathrm{p}<0,01)$, lo que equivale a un aumento relativo de $11 \%$ (Figura 6). Sin embargo, el efecto fue mayor en pacientes con indicación clásica de terapia de TRC, es decir, aquellos en ritmo sinusal con BCRI, FEVI < 35\% y CF III-IV, en que se observó una mejoría de $32 \%$ (desde 25,5 + 5,9\% hasta $33,8 \pm 8,0 \%, \mathrm{p}<0,01)$.

De los pacientes portadores de CDI, 15 (19\%) han tenido descargas apropiadas.

\section{Sobrevida}

Durante el período de estudio 37\% de los pacientes falleció, con una sobrevida global de $86 \%$ a 1 año y $51 \%$ a 5 años (Figura 7). La principal causa de muerte fue la cardiovascular (77\%), obedecien-

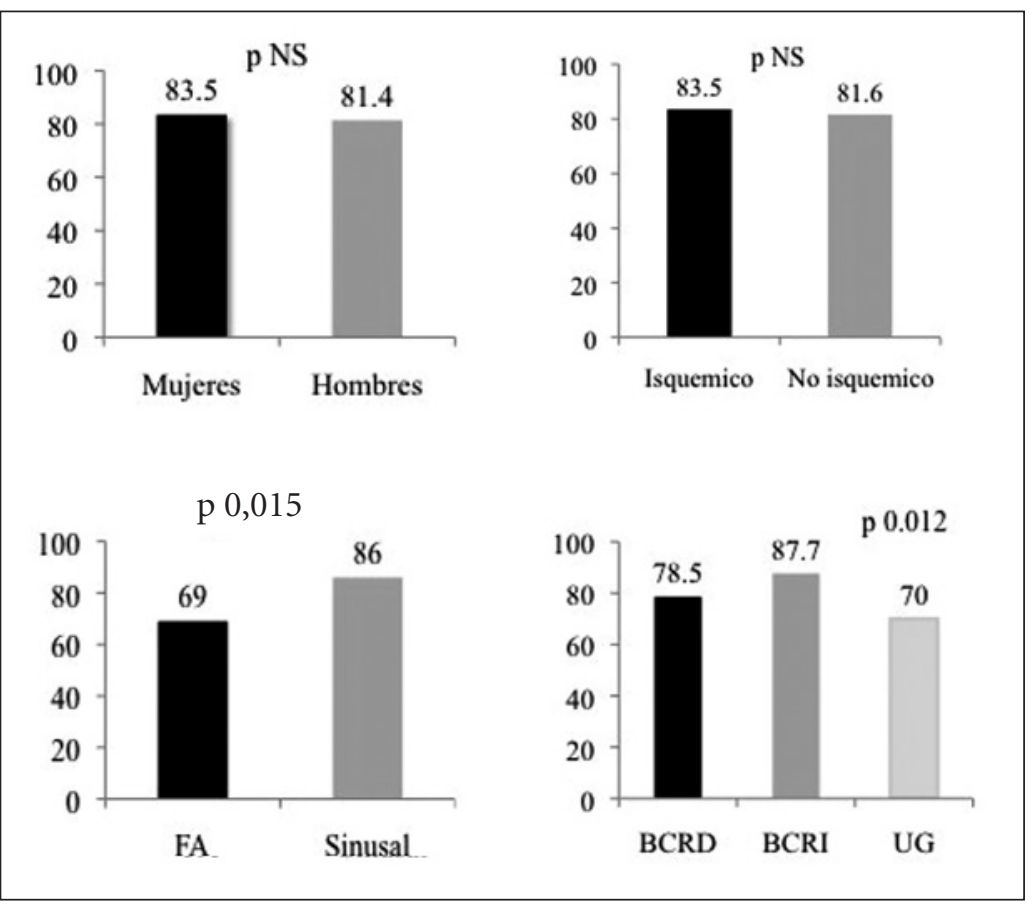

Figura 4. Tasa de respuesta clínica según subgrupos de pacientes. FA: BCRD: Bloqueo completo de rama derecha. BCRI: Bloqueo completo de rama izquierda. UG: Upgrade. 


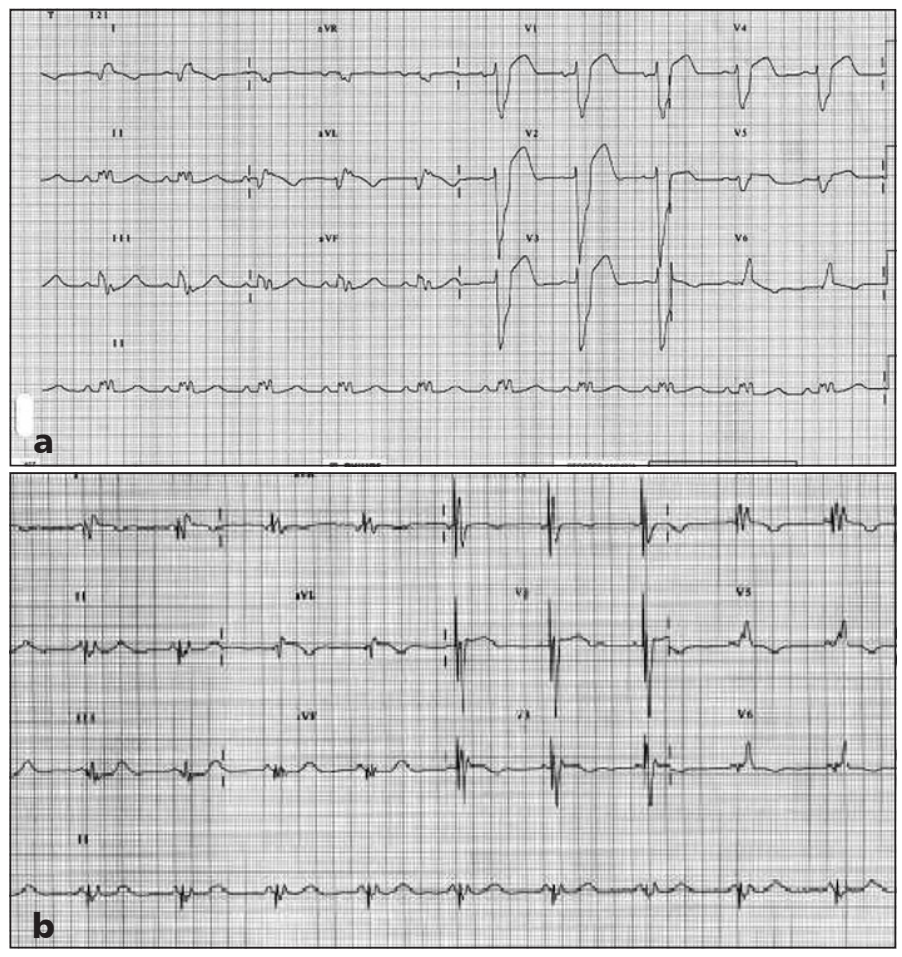

Figura 5. Electrocardiograma previo (a) e inmediatamente después de instalado el marcapaso resincronizador, donde se observa el artefacto de la estimulación biventricular (b). Se trata de un paciente con secuela de infarto de pared anterior, con bloqueo completo de rama izquierda. Posterior al implante de los electrodos ventriculares en la región septoinfundibular del ventrículo derecho y en una rama posterolateral del seno coronario, se observa una marcada disminución de la duración del QRS, con aumento de la amplitud de onda $R$ en las precordiales más derechas, como es esperable para la estimulación biventricular. do en su mayoría a progresión de la IC (Figura 8) y en segundo lugar a muerte súbita.

La sobrevida no difirió significativamente entre hombres y mujeres. Como puede apreciarse a partir de las curvas de sobrevida (Figura 9), fueron predictores de mortalidad la etiología isquémica (HR 1,48; p 0,05), la CF IV al momento del implante (HR 2,2; p < 0,01), el BCRD (HR 3,1; $\mathrm{p}<0,01)$ y la presencia de FA permanente (HR $3,4 ; p<0,001)$. La mortalidad no fue distinta en los pacientes que recibieron un resincronizador aislado (CRT-P) versus aquellos que además recibieron un CDI (CRT-D) (Figura 8), pese a que ambos grupos eran comparables en cuanto a edad, función sistólica VI y etiología. Tampoco se observó diferencia significativa en pacientes

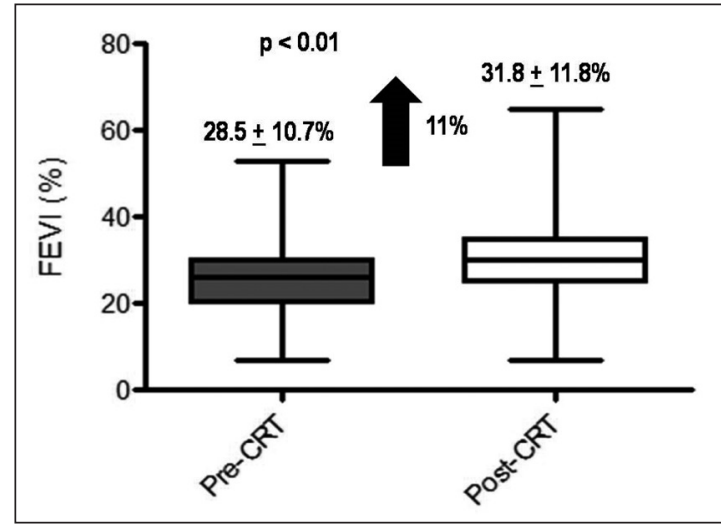

Figura 6. Fracción de eyección del VI por Método de Simpson (FEVI) pre y post implante de resincronizador.

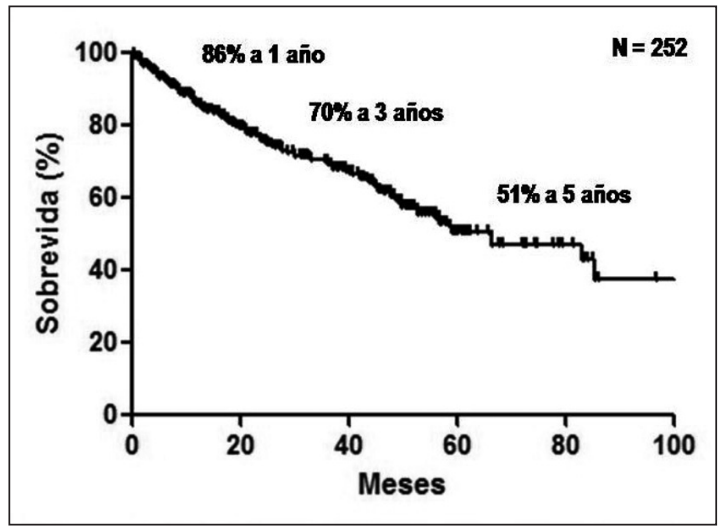

Figura 7. Sobrevida global. 


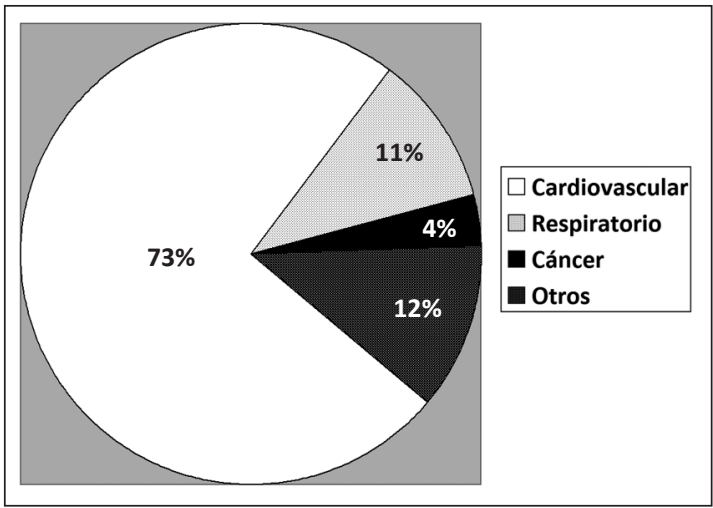

Figura 8. Causas de muerte. con disfunción VI severa respecto a aquellos con FEVI $>30 \%$.

\section{Discusión}

La presente serie de pacientes sometidos a TRC corresponde a la más grande publicada en nuestro país hasta la fecha y, en términos globales, reproduce en nuestro medio cifras similares a las grandes series internacionales ${ }^{12}$, con $93 \%$ de éxito en el implante.

La tasa de respuesta clínica observada en nuestros pacientes es mayor a la reportada en

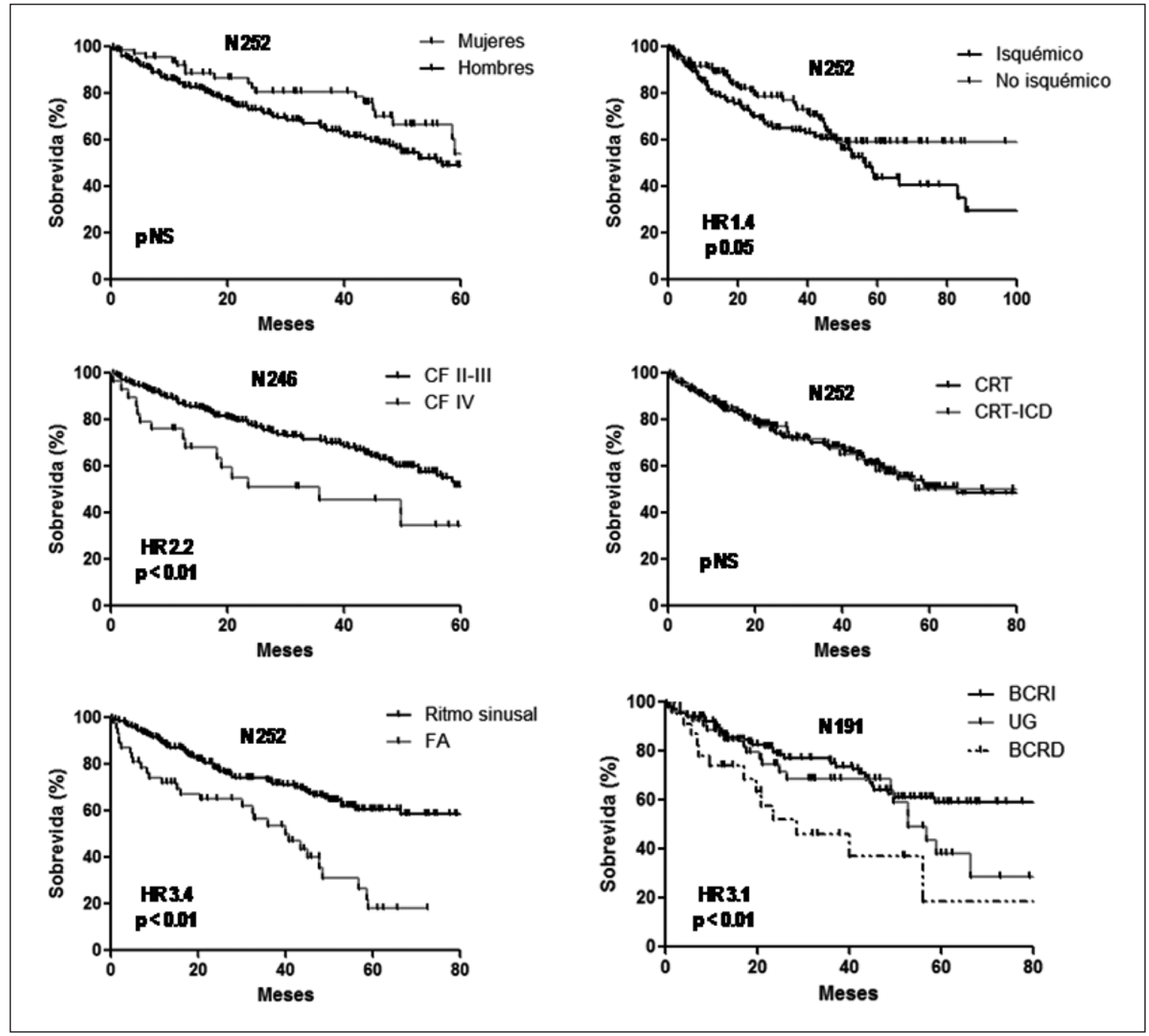

Figura 9. Curvas de sobrevida según subgrupos de pacientes. BCRD: Bloqueo completo de rama derecha. BCRI: Bloqueo completo de rama izquierda. UG: Upgrade. 
los estudios aleatorizados (60-70\%) y es similar a los reportes de estudios observacionales, que mostraron mejoría en al menos una categoría de CF en el 63-82\%. Es destacable además la baja tasa de mortalidad en nuestra serie, que si bien carece de un grupo control, es inferior a la esperada para pacientes en CF III-IV. Las 2 causas de muerte más frecuentes fueron, como es esperable, la progresión de la IC y la muerte súbita. Es llamativo no encontrar diferencias de sobrevida entre pacientes con y sin CDI, pese al 20\% de descargas apropiadas y a que ambos grupos eran comparables. Esto puede deberse a que la resincronización por sí sola es capaz de reducir la mortalidad global, como lo demostró el CARE-HF, y es probable que nuestra muestra haya sido insuficiente para observar las diferencias de sobrevida atribuibles al cardiodesfibrilador.

Las características generales de nuestros pacientes son comparables a las series internacionales $^{12}$, aunque existen algunas diferencias importantes. Así por ejemplo, los estudios con distribución aleatoria han excluido sistemáticamente a pacientes en FA, que en nuestra serie correspondían a 1 de cada 5 sujetos. Esta cifra es comparable a la práctica rutinaria mundial, como lo demuestra el ESC CRT Survey ${ }^{13}$, en que 23\% de los pacientes se encontraba en FA. En nuestra serie la presencia de FA se asoció a una menor tasa de respuesta y a un riesgo de mortalidad 3 veces mayor. Si bien la evidencia disponible para pacientes en FA es de menor extensión y calidad que en pacientes en ritmo sinusal, existe evidencia derivada de varios estudios observacionales pequeños ${ }^{14,15}$ y un metanálisis ${ }^{16}$ de que los pacientes con FA experimentan mejoría de la función VI y la capacidad de ejercicio, que es comparable a la alcanzada en pacientes en ritmo sinusal. Esto último difiere de lo observado en nuestra serie. Un registro prosprectivo extenso ${ }^{17}$ y una revisión sistemática ${ }^{18}$ mostraron que los pacientes con IC y FA tratados con un marcapaso resincronizador obtuvieron el mismo beneficio en sobrevida que los pacientes en ritmo sinusal sólo cuando el implante del resincronizador se asoció a ablación del nodo $\mathrm{AV}$, procedimiento complementario realizado en 3/4 partes de nuestros pacientes.

Asimismo, 25\% de los pacientes en nuestra serie correspondía a individuos con implante previo de un dispositivo (VVI o DDD), otro grupo excluido de los estudios aleatorios. Sa- bemos que la estimulación ventricular derecha pura genera un patrón de activación ventricular asincrónico ${ }^{19}$. Estudios animales han mostrado que la estimulación derecha a largo plazo produce desorden de las miofibrillas, hipertrofia ventricular asimétrica y dilatación del $\mathrm{VI}^{20-22}$. En humanos, estudios ecocardiográficos confirman la activación asincrónica y eficiencia ventricular reducida frente a la estimulación derecha pura, y otros han demostrado elevación de marcadores de IC y de estrés ${ }^{23-25}$. Estudios a gran escala, como el MOST y el DAVID han determinado que este tipo de estimulación es deletérea en términos de síntomas de IC, por lo que actualmente se trata de evitar estimulación ventricular derecha innecesaria ${ }^{26,27}$. Estudios clínicos relativamente pequeños han mostrado que en pacientes con IC y estimulación ventricular derecha, el "upgrade" a un sistema biventricular resulta beneficioso ${ }^{28,29}$. En este mismo sentido, se ha postulado que en pacientes que requieren estimulación ventricular permanente, la estimulación biventricular podría prevenir los efectos adversos de la estimulación derecha pura.

El 13\% de los pacientes correspondía a individuos con IC leve (NYHA I-II). La TRC en este subgrupo de pacientes fue abordado por los estudios MADIT-CRT ${ }^{10}$ y REVERSE ${ }^{11}$. En conjunto, ambos estudios incluyeron 2.430 pacientes en clase NYHA I o II y los hallazgos incluyeron mejoría del estatus funcional, disminución de las hospitalizaciones por IC y remodelado cardiaco reverso. Por su parte, el RAFT mostró que en pacientes con IC moderada (NYHA II-III) la adición de un CRT a un ICD se asoció a menos muertes y hospitalizaciones por IC $^{30}$.

Por corresponder al análisis retrospectivo de una serie clínica y no a un estudio prospectivo, una limitación importante de este estudio es la falta de evaluaciones sistematizadas objetivas para todo nuestro universo. Si bien el beneficio clínico fue evaluado en forma subjetiva, en la mayoría de los casos éste fue categórico y sostenido en el tiempo, lo que hace improbable que un efecto placebo juegue un rol importante en los resultados favorables observados.

\section{Conclusión}

Los resultados de esta serie correspondiente a la "vida real" revelan una alta tasa de respuesta 
clínica, comparable a la observada en los estudios aleatorios. A nuestro juicio, para lograr los efectos benéficos esperables de la terapia de resincronización constituyen elementos esenciales una correcta selección de los pacientes, la adecuada posición del electrodo VI (en general en una rama posterolateral o lateral, evitando posiciones anteriores), el asegurar una alta tasa de estimulación biventricular y la práctica de optimizar los intervalos auriculoventricular e interventricular.

En nuestra serie la progresión de la falla cardiaca fue la causa principal de muerte y fueron predictores de peor sobrevida la etiología isquémica, CF IV, BCRD y la presencia de FA. Es interesante no haber encontrado diferencias en la sobrevida de los pacientes con y sin cardiodesfibrilador, lo que podría ser relevante para la toma de decisiones en países que, como el nuestro, cuentan con recursos de salud limitados.

\section{Referencias}

1. Dayer M, Cowie MR. Heart failure: diagnosis and healthcare burden. Clin Med 2004; 4 (1): 13-8.

2. Cleland JG, Daubert JC, Erdmann E, Freemantle N, Gras D, Kappenberger L, et al. The effect of cardiac resynchronization on morbidity and mortality in heart failure. $\mathrm{N}$ Engl J Med 2005; 352 (15): 1539-49.

3. Baldasseroni S, Opasich C, Gorini M, Lucci D, Marchionni N, Marini M, et al. Left bundle-branch block is associated with increased 1-year sudden and total mortality rate in 5517 outpatients with congestive heart failure: a report from the Italian network on congestive heart failure. Am Heart J 2002; 143 (3): 398-405.

4. Cazeau S, Leclercq C, Lavergne T, Walker S, Varma C, Linde $\mathrm{C}$, et al. Effects of multisite biventricular pacing in patients with heart failure and intraventricular conduction delay. N Engl J Med 2001; 344 (12): 873-80.

5. Auricchio A, Stellbrink C, Sack S, Block M, Vogt J, Bakker P, et al. Pacing Therapies in Congestive Heart Failure (PATH-CHF) Study Group. Long term clinical effect of hemodynamically optimized cardiac resynchronization therapy in patients with heart failure and ventricular conduction delay. J Am Coll Cardiol 2002; 39 (12) 2026-33.

6. Abraham WT, Fisher WG, Smith AL, Delurgio DB, Leon AR, Loh E, et al. MIRACLE Study Group. Multicenter InSync Randomized Clinical Evaluation. Cardiac resynchronization in chronic heart failure. N Engl J Med 2002; 346 (24): 1845-53.

7. Young JB, Abraham WT, Smith AL, Leon AR, Lieberman
R, Wilkoff B, et al. Multicenter InSync ICD Randomized Clinical Evaluation (MIRACLE ICD) Trial Investigators. Combined cardiac resynchronization and implantable cardioversion defibrillation in advanced chronic heart failure: the MIRACLE ICD Trial. JAMA; 289 (20): 2685 94.

8. Higgins SL, Hummel JD, Niazi IK, Giudici MC, Worley SJ, Saxon LA, et al. Cardiac resynchronization therapy for the treatment of heart failure in patients with intraventricular conduction delay and malignant ventricular tachyarrythmias. J Am Coll Cardiol 2003; 42 (8): 1454-9.

9. Bristow MR, Saxon LA, Boehmer J, Krueger S, Kass DA, De Marco T, et al. Comparison of medical therapy, Pacing, and Defibrillation in Heart Failure (COMPANION) Investigators. Cardiac resynchronization therapy with or without an implantable defibrillator in advanced chronic heart failure. N Engl J Med 2004; 350 (21): 2140-50.

10. Moss AJ, Hall WJ, Cannom DS, Klein H, Brown MW, Daubert JP, et al. Cardiac-resynchronization therapy for the prevention of heart-failure events. N Engl J Med 2009; 361 (14): 1329-38.

11. Linde C, Abraham WT, Gold MR, St John Sutton M, Ghio S, Daubert C. Randomized trial of cardiac resynchronization in mildly symptomatic heart failure patients and in asymptomatic patients with left ventricular dysfunction and previous heart failure symptoms. J Am Coll Cardiol 2008; 52 (23): 1834-43.

12. McAlister FA, Ezekowitz J, Hooton N, Vandermeer B, Spooner C, Dryden DM, et al. Cardiac Resynchronization Therapy for Patients with Left Ventricular Systolic Dysfunction. A Systematic Review. JAMA 2007; 297 (22): 2502-14.

13. Dickstein K, Bogale N, Priori S, Auricchio A, Cleland JG, Gitt A, et al. The European cardiac resynchronization therapy survey. Eur Heart J 2009; 30 (20): 2450-60.

14. Khadjooi K, Foley PW, Chalil S, Anthony J, Smith RE, Freeneaux MP, et al. Long-term effects of cardiac resynchronisation therapy in patients with atrial fibrillation. Heart 2008; 94 (7): 879-83.

15. Delnoy PP, Ottervanger JP, Luttikhuis HO, Elvan A, Misier AR, Beukema WP, et al. Comparison of usefulness of cardiac resynchronization therapy in patients with atrial fibrillation and heart failure versus patients with sinus rhythm and heart failure. Am J Cardiol 2007; 99 (9): 1252-7.

16. Upadhyay GA, Choudhry NK, Auricchio A, Ruskin J, Singh JP. Cardiac resynchronization in patients with atrial fibrillation: a meta-analysis of prospective cohort studies. J Am Coll Cardiol 2008; 52 (15): 1239-46.

17. Gasparini M, Auricchio A, Metra M, Regoli F, Fantoni 
C, Lamp B, et al. Long-term survival in patients undergoing cardiac resynchronization therapy: the importance of performing atrio-ventricular junction ablation in patients with permanent atrial fibrillation. Eur Heart J 2008; 29 (13): 1644-52.

18. Ganesan A, Brooks A, Roberts-Thomson KC, Lau DH, Kalman J, Sanders P. Role of AV nodal ablation in cardiac resynchronization therapy in patients with coexistent atrial fibrillation and heart failure: a systematic review. J Am Coll Cardiol 2012; 59 (8): 719-26.

19. Wyman BT, Hunter WC, Prinzen FW, McVeigh ER. Mapping propagation of mechanical activation in the paced heart with MRI tagging. Am J Physiol 1999; 276: H881-H891.

20. van Oosterhout MF, Prinzen FW, Arts T, Schreuder JJ, Vanagt WY, Cleutjens, et al. Asynchronous electrical activation induces asymmetrical hypertrophy of the left ventricular wall. Circulation 1998; 98: 588-95.

21. Adomian GE, Beazell J. Myofibrillar disarray produced in normal hearts by chronic electrical pacing. Am Heart J 1986 (6); 112: 79-83.

22. Prinzen FW, Peschar M. Relation between the pacing induced sequence of activation and left ventricular pump function in animals. Pacing Clin Electrophysiol 2002; 25: 484-98.

23. Ichiki H, Okitani N, Hamasaki S, Ishida S, Kataoka T, Ogawa $\mathrm{M}$, et al. Effect of right ventricular apex pacing on the Tei index and brain natriuretic peptide in patients with dual chamber pacemaker. Pacing Clin Electrophysiol 2006; 29 (9): 985-90.

24. Al-Hesayen A, Parker JD. Adverse effects of atrioventricular synchronous right ventricular pacing on left ventricular sympathetic activity, efficiency, and hemodynamic status. Am J Physiol Heart Circ Physiol 2006; 291 (5): H2377-H2379.

25. Tops LF, Schalij MJ, Holman ER, van Erven L, van der Wall EE, Bax JJ. Right ventricular pacing can induce ventricular dyssynchrony in patients with atrial fibrillationafter atrioventricular node ablation. J Am Coll Cardiol 2006; 48 (8): 1642-8.

26. Sweeney MO, Hellkamp AS, Ellenbogen KA, Greenspon AJ, Freedman RA, Lee KL, et al. Adverse effect of ventricular pacing on heart failure and atrial fibrillation among patients with normal baseline QRS duration in a clinical trial of pacemaker therapy for sinus node dysfunction. Circulation 2003; 107 (23): 2932-7.

27. Wilkoff BL, Cook JR, Epstein AE, Greene HL, Hallstrom AP, Hsia $\mathrm{H}$, et al. Dual-chamber pacing or ventricular backup pacing in patients with an implantable defibrillator: the Dual Chamber and VVI implantable Defibrillator (DAVID) Trial. JAMA 2002; 288 (24): 3115-23.

28. Eldadah ZA, Rosen B, Hay I, Edvardsen T, Jayam V, Dickfeld $\mathrm{T}$, et al. The benefit of upgrading chronicle right ventricle-paced heart failure patients to resynchronization therapy demonstrated by strain rate imaging. Heart Rhythm 2006; 3 (4): 435-42.

29. Horwich T, Foster E, De Marco T, Tseng Z, Saxon L. Effects of resynchronization therapy on cardiac function in pacemaker patients "upgraded" to biventricular devices. J Cardiovasc Electrophysiol 2004; 15 (11): 1284-9.

30. Tang ASL, Wells G, Talajic M, Arnold MO, Sheldon R, Connolly S, et al. Cardiac-resynchronization therapy for mild to moderate heart failure. N Engl J Med 2010; 363 (25): 2385-95. 